\title{
Hybrid Actuators for Enhanced Automation in D\&D Remote Systems Tasks
}

\section{Research Objectives}

Revolutionary changes in both the design and control of manipulation systems are required to enable autonomous operations in unstructured environments, as those defined for D\&D tasks. Many researchers are exploring issues associated with the control of existing manipulation systems, but there is very little research effort directed towards enabling technologies that will provide significant improvement in the mechanical performance of these systems. Neither advanced controls or improved mechanical performance alone will enable a revolutionary new class of manipulation systems. The best control algorithms will not expand the performance of an actuated system beyond its physical limitations. The focus of this research is to explore advanced actuation methodologies that have the performance and capacity required for a revolutionary new class of manipulation systems that will enable autonomous operations in D\&D environments. There are three fundamental goals associated with achieving the required breakthroughs in actuation technology.

1) To understand the basic science associated with a new type of piezoelectric ceramic material and understand the engineering science associated with utilizing this smart material for a new type of actuator. While piezoelectric ceramic materials have traditionally been utilized as transducers, how one can utilize these materials for actuators is being explored. The focus of this project is on piezoelectric ceramic materials; however, the foundation that is being developed could also benefit research into magnetostrictive materials.

2) To study design issues associated with a hybrid actuator that has the power density of hydraulic but functions like a conventional electric motor.

3) To provide the enabling technology to allow remote systems to automate many of the subtask associated with D\&D applications and to facilitate tetherless operations.

\section{Research Progress and Implications}

This section summarizes work after 9 months of a 3-year project. The general models of the piezoelectric crystal and the mechanical pump are about completed. The dominate nonlinearities, such as the mechanical load model of the hydraulic pump, are being developed in detail. Simulink models (Simulink is a commercial simulation package from MathWorks, Inc.) are in the process of being constructed. Understanding these models is critical to the research goals of this proposal.

A conventional PZT stack is being used in an experimental setup and data is being collected for various operating conditions. Variations in the electrical elements as a function of voltage and frequency have been taken and the results are being examined. One major observation has already been made concerning the effective capacitance of the piezoelectric stack. We observed significant variations in the material capacitance as a function of the applied voltage. The linear models of the piezoelectric stack don't demonstrate this phenomenon. Further, PZN-PT stack has been purchased and should 
arrive in late July. The models associated with the PZN-PT material will be compared to the conventional PZT stack.

The overall goal of the simulation effort is to see if the power densities needed are achievable based on measured values of the piezoelectric materials.

Modeling associated with Subtask 1.1 will be completed by the end of June. The major test stand in Subtask 1.2 has been constructed and the PZN-PT stack is on order. The verification of the models should be completed by the end of July and then Subtask 1.3 will start. Subtask 1.3 will incorporate these updated models and then a simulation will be conducted to verify that the feasibility of the actuator being proposed is possible based on the power levels and power densities specified in the proposal.

\section{Planned Activities}

For this fiscal year, finishing up the models and taking the remaining measurements of the PZT stack will be completed by July. The PZN-PT stack should be finished up in August along with the overall computer simulation demonstrating the feasibility of the overall concept. Everything is on schedule.

For the next fiscal year (FY03), small distributive valves, which possess the property of being very responsive (possible $2 \mathrm{kHz}$ ), will be examined, modeled, and tested. An experimental setup will be used to verify that the fluid dynamic forces on the valves match with the computer simulations. Passive valves will be initially examined. The next step would be to determine if the actual measured powered densities and simulated power densities match at different operating frequencies. This will require a careful design of the mechanical valves and how they interact with the load and the piezoelectric stack. The pumping chamber and the valves will be assembled together. Careful measurements and comparisons to the mathematical modeled will be made. Utilizing conventional power electronics for piezoelectric actuators, a working pump will be demonstrated (i.e., without the final power electronics). A power density of 6.5 Watts $/ \mathrm{cm}^{3}$ for the piezoelectric stack is a target goal.

For the final fiscal year (FY03), after all the models for the piezoelectric actuator and pump have been developed, the overall system will be simulated. At this point, a series resonant power converter will be simulated and its overall performance evaluated. The challenge will be designing a robust controller that will handle large variations in both load and plant parameters. Traditional resonate converters are designed for fixed loads. Feedback of both voltage and frequency will be utilized to compensated for load and plant variations. We will evaluate the overall sensitivity of the switching topology and compensation network. As a proof-of-principle, the power electronics will be assembled, tested, and compared to the simulation. An integrated actuator will be constructed and tested.

\section{Information Access}

None at this time. 